\title{
Analysis on the Influence of Transmission Lines span on Passive Interference in Shortwave
}

\author{
Lu Ying ${ }^{1}$, Zhao Zhibin, Zhang Jian gong ${ }^{2}$ and Gan Zheyuan ${ }^{2,1}$ \\ ${ }^{1}$ College of Electrical and Electronical, North China Electric Power University, Beijing 102206, China \\ ${ }^{2}$ China Electric Power Research Institute, Wuhan, China
}

\begin{abstract}
The passive interference of transmission lines to nearby radio stations may affect the effective reception and transmission of radio station signals. Therefore, the accurate calculation of the electromagnetic scattering of transmission lines under the condition of external electromagnetic waves is the basis for determining the reasonable avoidance spacing of the two. For passive stations operating in shortwave frequencies, passive interference is mainly generated by the tower, and span is one of the most significant factors affecting passive interference. This paper uses the method of moments to carry out the passive interference calculations under normal circumstances, expounds the method of calculating the electromagnetic field of transmission line at the same time. And elaborates the method for calculating the electromagnetic field of the transmission line, obtains the space electric field intensity of the transmission line at the same working frequency and space location of the plane wave. Applying the approximate formula to calculate the formula for the span and critical distance between the observation point and the transmission line.
\end{abstract}

\section{Introduction}

In recent years, with the rapid development of science and technology, massive construction of infrastructure such as transmission lines and radio stations has led to a close distance between the two[1].In this case, even if the transmission line is not energized, its huge metal structure will affect the reception or transmission of signals from nearby radio stations[2-3]. This effect caused only by the scattering of the metal conductors of the transmission line is called passive interference. How to determine the change of the avoidance distance between the two due to the different transmission span is the key to the consideration of power line construction.

In response to this problem, the United States[46],Canada[7],Japan[8,9] and other countries have carried out related research on the influence of power lines on signals in different frequency bands such as MF, VHF/UHV, and $\mathrm{m}$ waves since 1960s. The research object involves radio wide broadcast[7], radar navigation[6] and other fields[8]. Since the construction of UHV transmission lines in China, China Electric Power Research Institute Wuhan Branch, North China Electric Power University, and the China Three Gorges University have conducted a systematic and in-depth research on the electric field strength characteristics of UHV transmission lines, and proposed the avoidance standard for $1000 \mathrm{kV}$ AC transmission lines.

In recent years, with the gradual improvement of the voltage level of transmission lines in China and the continuous construction of UHV AC and DC transmission lines, the impact Of UHV transmission lines on passive interference of radio stations has become increasingly serious.[10-11] At present, the electromagnetic radiation problem of transmission lines to radio facilities has become one of the most concerned priorities of various departments. According to the correlation and correlation analysis of the electric field strength curves of the short-wavelength transmission lines based on various single macroscopic structures, it can effectively explain that the influence of the range on the passive interference resonance phenomenon in the short-wave band is most significant, that is, from the perspective of macrostructure. the span is the decisive influence factor[8] of the passive interference of the transmission line in the short-wave band. Therefore, in the short-wave frequency range, the study of the influence of the span on passive interference is the key to study the electromagnetic radiation generated by the transmission line in the short-wave frequency.

\section{Modeling}

\subsection{Principle analysis}

The passive interference generated by the transmission line is mainly caused by the electromagnetic radiation of the iron tower, which is related to the frequency, polarization mode and relative position of the affected signal. Therefore, the frequency of the electromagnetic wave is set to a certain frequency point, and the 
polarization mode is vertical polarization. According to the previous article, the influence of the span on the passive interference resonance phenomenon in the shortwave band is most significant..

The effects of electromagnetic interference are: $S=20 \log \frac{E_{\mathrm{Y}}}{E_{N}}$

$E_{Y}$ indicates that the transmission line and the impact of the tower affect the spatial electric field strength of the observation point; ${ }^{E_{N}}$ indicates that the transmission line and the tower affect the spatial electric field strength of the previous observation point.

\subsection{Tower type selection and parameters}

Since there is no relevant standard reference, the typical tower type of the horn tower in the AC transmission line is used in calculation. The height of the tower is $98.5 \mathrm{~m}$, the structure and parameters of the AC transmission line tower are shown in Figure 1.

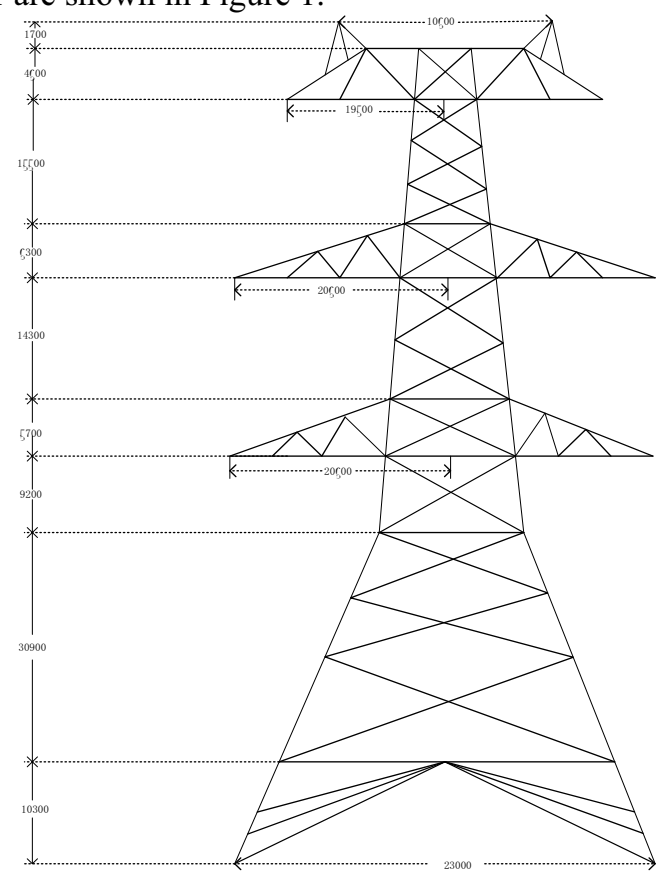

Fig.1 Outline dimension of tower type

\section{Comparison of calculation results of spatial electric strength}

\section{1. principle basis}

In the paper of "Research on Passive Interference of UHV AC Transmission Lines to adjacent Stations", the mutual coupling effect between towers is discussed[8]. Since the mutual interference between the towers cannot ignored, here two towers are taken as an example. The electromagnetic interference coupling model as shown in Fig. 2 is established. The two towers are respectively regarded as a transmitting antenna and a receiving antenna, and the output power of the transmitting antenna port is $\boldsymbol{P}_{t}$, the input power of the transmitting antenna port is $\boldsymbol{P}_{r}$

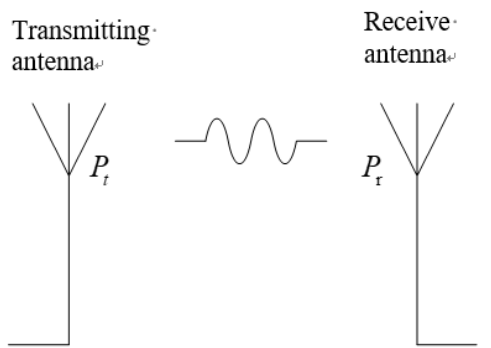

Fig.2 Radiative interference coupling model between two antennas

Define the coupling degree c between the far-field antenna as:

$$
c=\frac{\boldsymbol{P}_{r}}{\boldsymbol{P}_{t}}
$$

Fig. 3 is a schematic diagram showing the relative positions of the off-angles between the transmitting and receiving antennas. ${ }^{G_{r}}$ and ${ }^{G_{t}}$ :the gain of the receiving and transmitting antenna; $\theta_{1}$ and $\theta_{2}$ :the direction in which the radiation direction of the two antenna deviate from each other by the main beam of the opposite party; $\varphi_{1}$ and $\varphi_{2}$ :the angle between the main radiation direction of the two antennas and the horizontal plane; $\lambda$ :the working wavelength; $S$ :a polarization matching coefficient between the transmitting antenna and the receiving antenna; $L$ :the shortest distance between the two antennas.

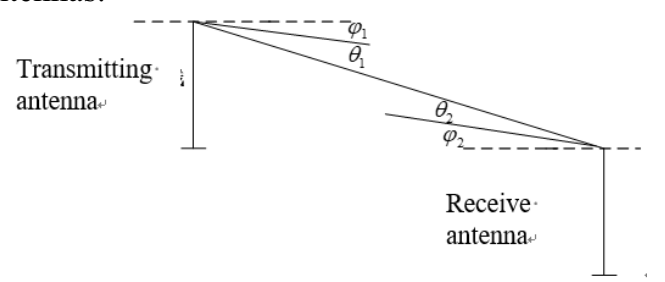

Fig.3 Mutual departure angle between transmitting and receiving antennas

So there are:

$$
P_{r}=\frac{\cos \theta_{1} \cos \theta_{2} P_{t} G_{t} G_{r} \lambda^{2} S}{(4 \pi L)^{2}}
$$

Finished up:

$$
c=\frac{\cos \theta_{1} \cos \theta_{2} G_{t} G_{r} \lambda^{2} S}{(4 \pi L)^{2}}
$$

Idealize all the parameters and organize them :

$$
c=\left(\frac{\lambda}{4 \pi d}\right)^{2}
$$

Equation (3)can be derived into the array, only need to $d$ rewritten into $d_{i j}$. It can be regarded as the interference coupling coefficient of the $\mathrm{i}$-th transmit antenna to the $\mathrm{j}$-th receive antenna, which has the following from: 


$$
C=\left\{\begin{array}{l}
\left(\frac{\lambda}{4 \pi d_{11}}\right)^{2}\left(\frac{\lambda}{4 \pi d_{12}}\right)^{2} \ldots \ldots\left(\frac{\lambda}{4 \pi d_{1 n}}\right)^{2} \\
\left(\frac{\lambda}{4 \pi d_{21}}\right)^{2}\left(\frac{\lambda}{4 \pi d_{22}}\right)^{2} \ldots \ldots\left(\frac{\lambda}{4 \pi d_{2 n}}\right)^{2} \\
\left(\frac{\lambda}{4 \pi d_{n 1}}\right)^{2}\left(\frac{\lambda}{4 \pi d_{n 2}}\right)^{2} \ldots \ldots\left(\frac{\lambda}{4 \pi d_{n n}}\right)^{2}
\end{array}\right.
$$

The physical meaning of the elements on the diagonal is the degree of autotransformation of a single antenna element itself, and its own coupling to itself is 1 , so matrix $\mathrm{C}$ can be rewritten into

$$
C=\left\{\begin{array}{l}
1 \quad\left(\frac{\lambda}{4 \pi d_{12}}\right)^{2} \ldots \ldots\left(\frac{\lambda}{4 \pi d_{1 n}}\right)^{2} \\
\left(\frac{\lambda}{4 \pi d_{21}}\right)^{2}\left(\frac{\lambda}{4 \pi d_{22}}\right)^{2} \ldots \ldots\left(\frac{\lambda}{4 \pi d_{2 n}}\right)^{2} \\
\left(\frac{\lambda}{4 \pi d_{n 1}}\right)^{2}\left(\frac{\lambda}{4 \pi d_{n 2}}\right)^{2} \ldots \ldots \ldots 1
\end{array}\right.
$$

Taking into account the coupling interference coefficient between the arrays, the electric field strength of the i-th tower is multiplied by the mutual coupling interference coefficient with respect to the electromagnetic radiation field strength of the 0th tower to the far point. Vertically polarized plane wave incident angle is $\alpha . E_{i}$ is the Field strength for the i-th tower at the required far-field point; $r_{0}, r_{i}$ the distance between the 0 th and $\mathrm{i}$-th towers to the required far field point 。

$$
\begin{gathered}
E_{i}=\left|E_{0}\right| \bullet \frac{r_{0}}{r_{i}} e^{j\left(\frac{2 \pi\left(r_{i}-r_{0}\right)}{\lambda}-\frac{2 \pi i * d \sin \alpha}{\lambda}\right)} \\
=\left|E_{0}\right| \bullet \frac{r_{0}}{r_{i}} e^{j \frac{2 \pi\left(r_{i}-r_{0}-i * d \sin \alpha\right)}{\lambda}} \\
E_{\text {all }}=E_{i} \bullet C
\end{gathered}
$$

The method of using the electromagnetic field strength of a single tower as a $\mathrm{t}=$ reference and multiplying the array influence coefficient to the obtain the far-field characteristics is accurate and effective from the perspective of simulation calculation. This method can greatly improve the calculation efficiency.

\subsection{Electric field strength calculation results}

In order to study the influence of transmission line span in passive interference, this paper compared the electric field strength generated by different transmission lines with different spans.as the same time, in order to ensure the universality of the results, this section sets different frequencies. Keep other factors constant.

\subsubsection{Analysis of result at a frequency of $5 \mathrm{MHz}$}

The calculated electric field strength of the transmission line in the short-wavelength band is plotted as a curve with the horizontal distance between the observation point and the transmission line(referred to as" horizontal distance" )and the range, as shown in Figure 4;figure 5(a)shows the Electric field intensity changes with horizontal distance; figure 5(b) Electric field intensity changes with the span. Figure 8 is the Critical distance between observation point and transmission line changes with the span(critical distance: a horizontal distance that ensures a monotonous decreasing trend of the electric field strength)

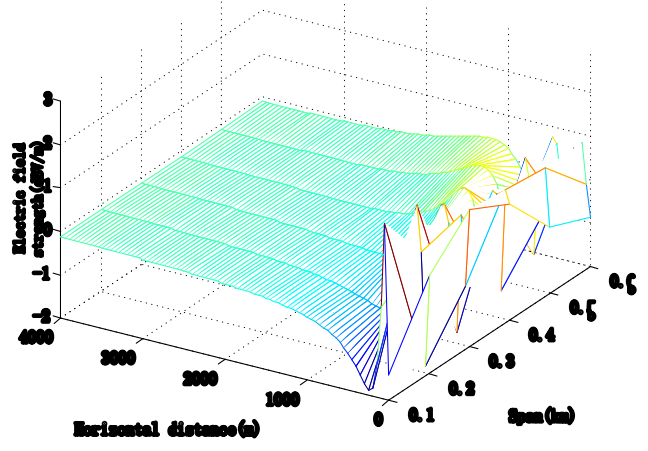

Fig.4 Electric field intensity changes with horizontal distance and the span

As can be seen from the above figure, as the span and horizontal distance increasing, the electric field strength level decreases. Indicating that the horizontal distance and the pan are important factors affecting the electric field strength.

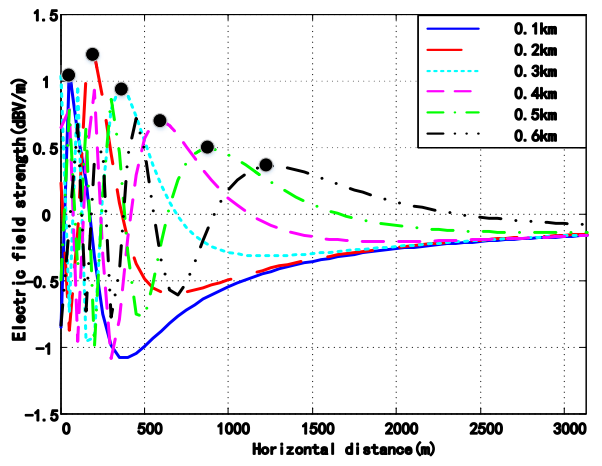

Fig.5(a) Electric field intensity changes with

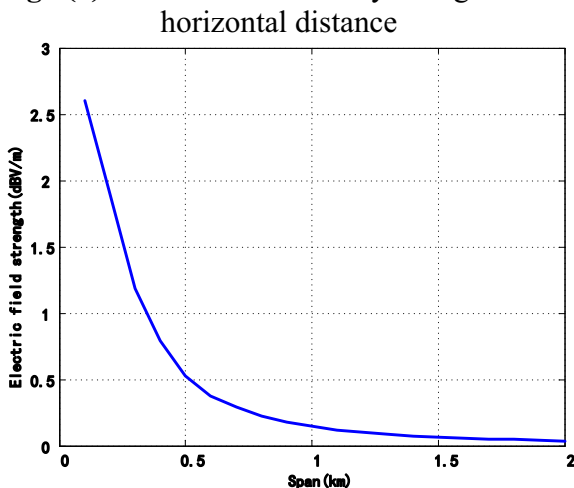

Fig.5(b) Electric field intensity changes with the span

In order to obtain the relationship between the critical distance and the gear distance more accurately, this section studies the towers of difference heights.

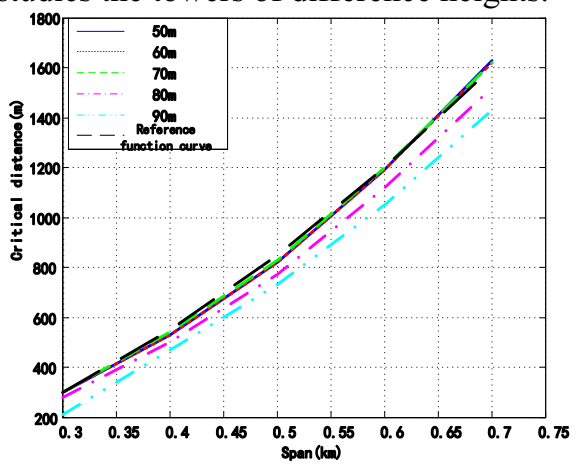


Fig.6 Critical distance between observation point and transmission line changes with the span

As shown in the figure, within the allowable range of error $(5 \%)$, the curve of the critical distance as a function of the span can be approximated by a function curve. The formula is as follows:

$$
L=\frac{d^{2}}{400}+\frac{3 d}{4}-150(d>0 k m)
$$

$L$ : ensuring the horizontal distance of the electric field strength in a monotonically decreasing trend, that is, the critical distance; $d$ :span。

It can be seen from the curve change of figure 7(a)and figure 7(b)that the critical distance is $L<1000 \mathrm{~m}$,or $d \geq 0.5 \mathrm{~km}$,Electric field strength $E<0.5 \mathrm{dBV} / m(S E<0.5)$, it can be considered that the influence of the electric field strength generated by the transmission line can be neglected. Therefore, the above horizontal distance and the span can be used as the basis for calculating the avoidance distance of the short-wave radio station and the transmission line.

\subsubsection{Calculation results at different frequencies}

In order to avoid the above results, this section have studied the results at different frequencies. Figure 7 is the electric field intensity level varies with the frequency.

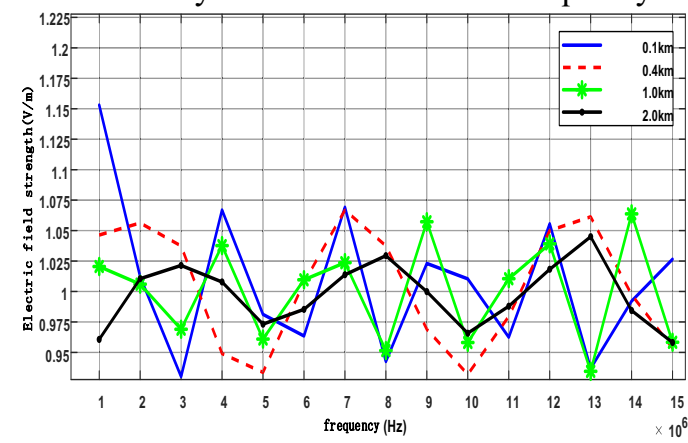

Fig.7 The electric field intensity level varies with the frequency

In order to ensures the universality of the research results, the frequency of the plane wave selected is $12 \mathrm{MHz}$. Figure $8(\mathrm{a})$ is the electric field intensity changes with horizontal distance; Figure $8(\mathrm{~b})$ is the electric field intensity level varies with the span; and Figure 9 is Critical distance changes with the span

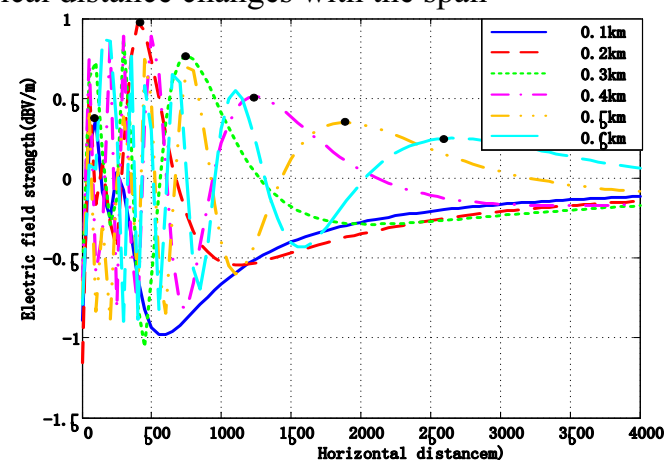

Fig.8(a) Electric field intensity changes with horizontal distance

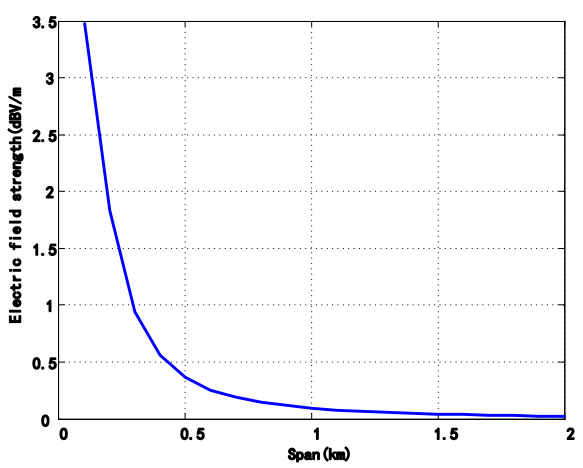

Fig.8(b) The electric field intensity level varies with the span

It can be seen from the curve change of Fig. 9 that as the span increases, the electric field strength decreases. And the results of this study are similar to the simulation results when the plane wave is $5 \mathrm{MHz}$

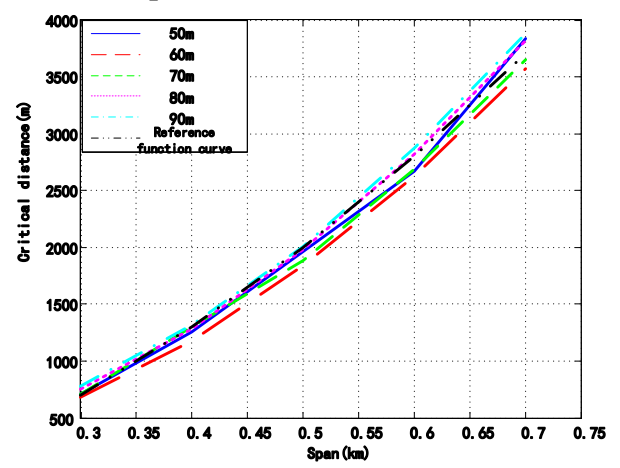

Fig.9 Critical distance changes with the span

It can be seen from the curve change of Fig. 11 that when the frequency of the plane wave is $12 \mathrm{MHz}$, the Critical distance changes with the span can be expressed by the following formula.

$$
L=2.4 *\left(\frac{d^{2}}{400}+\frac{3 d}{4}-150\right)(d>0 k m)
$$

In this case, if the range of the span is $0.3 \mathrm{~km} \sim 0.6 \mathrm{~km}$, only the critical distance of $1320 \mathrm{~m}$ is required to ensure the communication quality of the radio station.

This section also simulates the curve of the critical distance with the span when the frequency is $7 \mathrm{MHz}$ and $25 \mathrm{MHz}$.
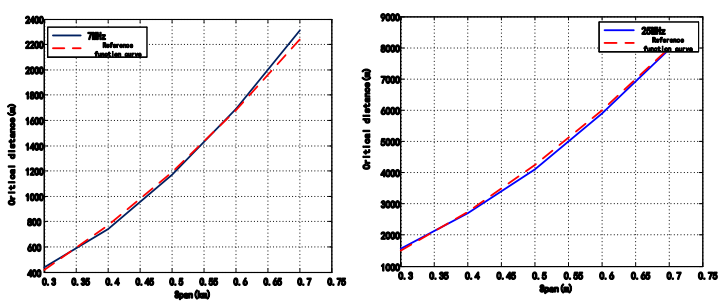

Fig.10 Maximum distance changes with the span with difference frequencies

The calculated fit curve is

$$
\begin{aligned}
& L=1.4 *\left(\frac{d^{2}}{400}+\frac{3 d}{4}-150\right)(d>0 k m) \\
& L=5 *\left(\frac{d^{2}}{400}+\frac{3 d}{4}-150\right)(d>0 k m)
\end{aligned}
$$


So, the relationship between the critical distance and the span can be expressed by the formula (13), and this formula is universal.

$$
L=\frac{\text { Frequency }}{5 \mathrm{MHz}} *\left(\frac{d^{2}}{400}+\frac{3 d}{4}-150\right)(d>0 \mathrm{~km})
$$

\section{3 conclusion}

The influence of transmission line span on passive interference is different through analysis in this paper. Derive the formula for calculating the distance between the span and the observation point and the transmission line, relationship between the tower height and the span can be summarized follows:

(1)This paper fits the relationship between the transmission line span and the critical distance, as shown in the following equation.

$$
L=\frac{\text { Frequency }}{5 M H z} *\left(\frac{d^{2}}{400}+\frac{3 d}{4}-150\right)(d>0 \mathrm{~km})
$$

This formula is verified by comparing the relationship between the actual span and the critical distance: In the short wave band, under the same tower height condition, if the incident frequency and the span of the plane wave are known, the above formula can be used to derive the critical distance at this time, which can predict the guard spacing more accurately when the range is different.

(2)In practical engineering, the distance between the span and the critical distance can be used to study the avoidance distance of transmission lines and radio stations. It greatly reduces the time required for modeling and improves the accuracy of simulation calculations.

\section{Acknowledgement}

This work was supported by the Project of SGCC (GY71-16-011)

\section{References}

1. Yin hui, Zhang xiaoming, Wang yantao, et a. Research on 3D visualization of electromagnetic interference for Ultra-high Voltage transmission lines. High Voltage Engineering.40,12 (2014)

2. Tang bo , Yang jiawei Huang li, et al. Guard spacing of the air surveillance radar station to Ultrahigh Voltage transmission line.40.4(2018)

3. Tang bo, Sun rui, Zhao xiaoming, et al. Passive interference solution for UHV transmission line in very high frequency band . Electric Power,49,10(2016)

4. Hill P C.Measurement of reradiation from lattice masts at V.H,F.[J].Proc Inst Elec Eng,111,12(1964)

5. Belroses J S,Lavrench W,Dunn J G,et al.The effects of reradiation from high rise buildings and transmission lines upon the radiation pattern of $\mathrm{MF}$ broadcasing antenna arrays[C]/Proceedings of AGARD/EPP Meeting .Spatind,Norway:[s.n.],1979.

6. Kamal Sarabandi, Moonsooo Park.A radar crosssection model for power lines at millmeter-wave frequency[J].IEEE Transactions on Antenna and Propagation,51,9(2003)

7. Truemen C W, Kubina S J. Scatting from power lines with the skywire insulated from the towers[J].IEEE Transactions on Broadcasting,40,2(1994)

8. Kinase. Theoretical analysis of reradiation characteristics of radio wave caused by transmission lines[ J] .NHK Technology Research, 20,3(1968)

9. Takeshita K,Takeshita S,Hashimoto H.Scattering characteristics of $\mathrm{VHF} / \mathrm{UHF}$ television broadcasting waves by overhead oower transmission conductors[J]..IEEE Transactions on Electromagnetic Compatibility,21,1(1979)

10. Chen bin , Tang bo, Cao hongying, et al. Association Analysis of Passive Interference Resonance Impact Factors for Transmission Lines at Short-Wave Frequency,36,6(2015

11. Gan zheyuna, Wang yanhai, Zhang jiangong, et al, Suppression of HF passive interference in transmission lines by using magnetic tube . High Voltage Engineering, 43,5(2017) 